\title{
Pour une expertise citoyenne des risques
}

\section{Gérard Lambert}

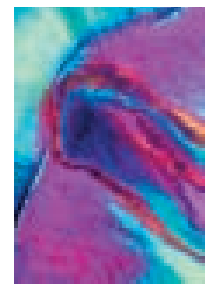

Éthers de glycol, amalgames dentaires, dioxines, mycotoxines, disrupteurs, téléphones portables, overdose de sel dans nos assiettes, André Cicollela et Dorothée Benoit-Browayes traquent les toxiques de la vie quotidienne. Le scientifique lanceur d'alerte et la journaliste spécialiste en santé environnementale signent un livre de référence sur les enjeux sanitaire de la société postindustrielle [1].

Les crises sanitaires et leurs lots de révélations aux parfums de scandale ont alerté l'opinion publique, inquiété les décideurs politiques et pointé les limites de l'évaluation scientifique. On serait en droit de penser que des affaires aussi retentissantes que celles du sang contaminé, de l'amiante ou de l'encéphalopathie spongiforme bovine ont suscité la création de réseaux de surveillance et d'expertise adaptés à la gestion des risques sanitaires. Mais, à en croire le livre d'André Cicollela et de Dorothée Benoit-Browaeys, nous en sommes encore loin. Beaucoup reste à faire, ne seraitce que pour prendre la juste mesure du danger, à défaut de vivre dans un monde réellement sécurisé. Car, aussi exemplaires qu'elles soient, ces affaires ne sont pas exceptionnelles. C'est l'un des enseignements que l'on tire de cet ouvrage solidement documenté, la reconnaissance d'une foule de risques environnementaux connus, identifiés, mais toujours pas réglés.

Alertes santé, c'est d'abord une formidable enquête sur ces dossiers que des citoyens, des scientifiques, des associations ont portés à bout de bras afin d'alerter le public et les autorités sur des risques méconnus. C'est l'histoire de ces hommes et des ces femmes qui, au nom de la santé publique, ont résisté aux pressions de leur hiérarchie, à l'arrogance d'experts englués dans des conflits d'intérêts et à la précarisation
Journaliste scientifique, 9, Villa Claude Monet, 75019 Paris, France.

glamb@noos.fr de leur situation professionnelle. S'ils affirment vouloir éviter la «victimisation» des lanceurs d'alerte, les auteurs n'évitent cependant pas l'écueil de les décrire comme des héros des temps modernes, des citoyens au sens plein du terme, des sentinelles dans la cité. On vibre avec Marc Edouard Colin, ce vétérinaire qui dénonce la toxicité du Gaucho ${ }^{\circledR}$ sous les calomnies du groupe Bayer, on s'inquiète pour la santé de Véronique Lapidès et des siens exposées aux polluants d'une ancienne usine Kodak et on voudrait venir en aide à Jean-Jacques Melet, qui se bat pied à pied pour éradiquer le mercure de nos cavités buccales. Le récit est précis et détaillé, chaque pièce versée au dossier dûment référencée, au point parfois de noyer le lecteur sous un flot d'acronymes et de rapports d'expertise.

Certains cas analysés pourront surprendre. Classé dans la seconde partie de l'ouvrage parmi les nouveaux risques, l'exemple de la légionellose traduit d'abord l'extraordinaire capacité d'adaptation de l'écologie bactérienne aux pratiques humaines. Et l'opiniâtreté de I'Institut de veille sanitaire ( $\ln V s)$, qui ne ménage pas ses efforts pour débusquer les tours aéroréfrigérantes contaminées, tend surtout à prouver que le lanceur d'alerte est en voie d'institutionnalisation. Autre cas inattendu, celui des brevets accordés à la société Myriad Genetics sur les gènes de prédisposition au cancer du sein, BRCA1 et BRCA2. La bataille juridique livrée par l'Institut Curie sous l'impulsion du Dr Dominique Stoppa-Lyonnet a abouti à la révocation de certains d'entre eux par l'Office européen des brevets. On mesure 
la portée de ce jugement qui fera jurisprudence, mais on $y$ voit plus une affaire de pratiques juridiques et de protection industrielle qu'une alerte santé. C'est que, selon les auteurs, la définition de la santé environnementale est large, puisqu'elle cherche à mettre en évidence «la responsabilité de tel ou tel facteur, physico-chimiques, ergonomique, social ou psychologique, dans l'atteinte de la santé ou dans le bon état de santé d'une personne ou d'une population ». La pratique sociale du brevet, qui stérilise la recherche scientifique sans garantir la qualité du diagnostic et des soins, entre donc dans la sphère de la santé environnementale.

Une autre qualité de cet ouvrage est de bousculer quelques idées reçues. Ainsi, l'impact de l'environnement sur la santé, qui est au centre de ce travail, remet la part héréditaire et génétique des maladies à sa juste place. L'expertise scientifique se voit démythifiée et rappelée à plus d'humilité en se positionnant comme une activité humaine dotée d'outils méthodologiques et techniques rationnels, mais jamais impartiale: «[...] il n'y a pas de savoir sans hypothèse préalable, donc sans projection d'une vision ». Enfin, le fait de s'adresser au public et de le considérer comme producteur de savoir fera frémir plus d'une bonne conscience emmurée dans ses certitudes.

Les auteurs ne s'en cachent pas, ils œuvrent pour la reconnaissance des risques sanitaires trop souvent négligés, occultés par les industriels et minimisés par des comités d'experts qui peinent à conserver leur indépendance. En matière de santé publique, soulignent-ils, les acteurs «doivent se contenter de bas niveaux de preuve pour mettre en place des plans de prévention qui, pour être efficaces, doivent s'appliquer à l'ensemble de la population». II faut donc prendre en compte les risques, et non le danger; «si vous faites le pari de l'épidémiologie et de la certitude scientifique, vous risquez de ne pas protéger les populations pendant tout le temps que vous menez les études, le temps de savoir si l'évaluation des risques est pertinente ou pas ». Dans la jungle des rapports contradictoires et des avis scientifiques divergents, il faudrait donc se ranger en priorité aux côtés du plus alarmiste, à condition bien sûr qu'il respecte les critères de bonnes pratiques. Et pourtant, que d'incertitudes dans l'évaluation du risque, un océan de doute qu'André Cicollela et Dorothée Benoit-Browaeys ne cherchent pas à dissimuler. Ce n'est pas un hasard si les maladies les plus souvent citées comme possiblement secondaires à une intoxication chronique sont celles dont les causes et la physiopathologie demeurent inconnues (maladies d'Alzheimer et de Parkinson, autisme, sclérose en plaques, lupus érythémateux disséminé, sclérodermie, une liste à laquelle on peut incorporer le cancer), ou des entités pathologiques dont la réalité est controversée (fibromyalgie, syndrome d'hyperactivité de l'enfant, syndrome d'hypersensibilité chimique multiple, syndrome des bâtiments malsains, perte de tolérance déclenchée par les toxiques...).

Cet ouvrage appelle à ouvrir un débat que pourrait nourrir chacune de ses pages. II décrit des problèmes concrets et fournit assez de preuves pour qu'il soit impossible de les nier. Mais il ne se limite pas à dresser un bilan. II ouvre des pistes intéressantes, à commencer par une proposition de loi pour la création d'un haut comité de l'expertise scientifique et la protection les lanceurs d'alerte. Une solution qui s'avérera fructueuse à la condition d'adopter la philosophie du Danish Board of Technology qui entend «combattre aussi bien les technophobies sans limite que la passion sans nuance pour la technologie ». $\diamond$

For a civic expertise of the risks

\section{RÉFÉRENCE}

1. Cicolella A, Benoit-Browaeys D. Alertes santé. Experts et citoyens face aux intérêts privés. Paris : Éditions Fayard, 2005.

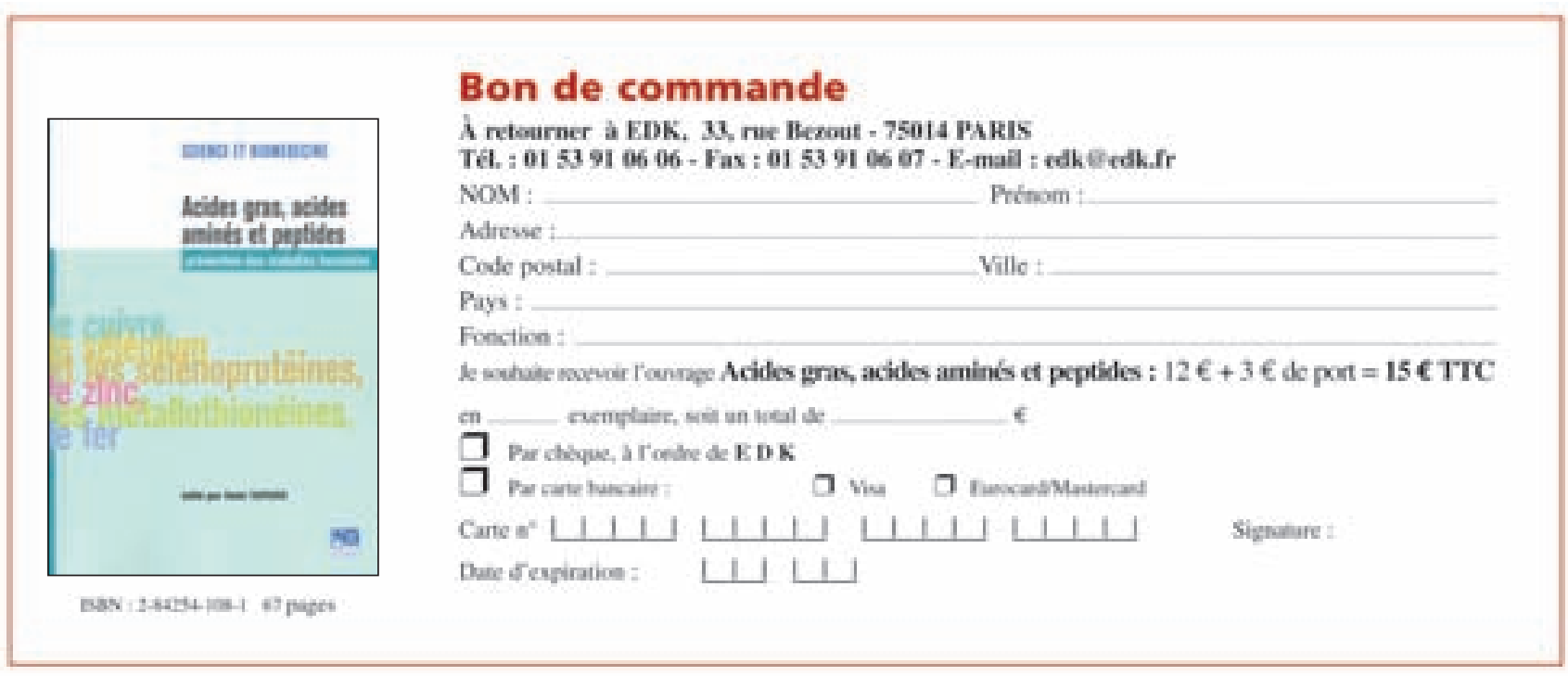

